\title{
Traits for depression related to agentic and external control
}

Santiago Castiello ${ }^{1,2}$, Salha Senan $^{3}$, Rachel M. Msetfi ${ }^{3,4,5}$ \& Robin A. Murphy ${ }^{1}$

${ }^{1}$ Department of Experimental Psychology, University of Oxford.

${ }^{2}$ University of Guadalajara

${ }^{3}$ Department of Psychology, King Abdulaziz University, Jeddah, Saudi Arabia

${ }^{4}$ Department of Psychology, University of Limerick, Republic of Ireland.

${ }^{5}$ Health Research Institute, University of Limerick, Republic of Ireland. 


\begin{abstract}
Depression has been linked to weakened perceptions of control. The experimental evidence derives from tasks with exposure to stable action-outcome contingencies. One assumption has been that performance represents a general cognitive bias that might manifest itself by a global performance difference. Another view is that people have specific situational perceptions of control reflecting their recent actions and the contingencies to which they are currently experiencing. In an experiment with $\mathrm{N}=$ 179, participants acquired one of four action-outcome sequences (Constant or Variable). We measured how learning was reflected in ratings of control and probability of responding in relation to mood. In three experimental treatments, the overall contingency across training involved an average moderate degree of control $(\Delta \mathrm{P}=0.25)$, but differed in how control varied (Constant or one of two Variable treatments). A fourth, control treatment involved a Constant zero degree of control $(\Delta \mathrm{P}=0.00)$. Participants rated their control before, during and after each sequence, providing measures of pre-existing bias, ratings of control in specific situations and generalised control perceptions. Specific control ratings were only influenced by the contingency experience and not pre-existing bias. Higher scores on the Beck's depression inventory were associated with weakened association between action and context ratings. Overall, these data suggest that human agency is related to rates of responding and that mood is related to a difference in sensitivity to the ratings of and responding to the context.
\end{abstract}


People with depression perceive their life events as uncontrollable. Patients report a sense of helplessness, reduced agency, whether or not they actually have such control (e.g., Abramson, Metalsky, \& Alloy, 1989; Seligman, 1975). This assertion is based, in part, on empirical findings involving people's ratings of specific situations in which the amount of available control is known and compared to control ratings. The comparison between the objective control and people's ratings of control allows inferences about symptomatic bias (Ackermann \& DeRubeis, 1991). An assumption underlying this work is that perceived uncontrollability in one specific situation generalises to other situations and even contributes to a generalised sense of control (learned helpless is a good example of this, e.g., Maier \& Seligman, 1976). It is unclear, however, how and whether specific control experiences combine to produce a generalised sense of control or whether generalised control is mainly determined by expectations, pre-existing bias and how responding to learn control (e.g., Blanco, Matute \& Vadillo, 2009). Here, we report the results of a study designed to test whether a generalised perception of control is influenced by levels of control that are available across situations, or by initial biases and expectations, and mood state. First however, we provide a brief empirical and theoretical background to this work.

Perceived control has been studied by measuring distinct aspects of the construct of control (for a review of constructs, see Skinner, 1996) here, we focus on contingency judgement and instrumental control, as these provide experimenters with a form of objective standard against which to evaluate people's perceptions, and evidence for bias (Ackermann \& DeRubeis, 1991; Dobson \& Franche, 1989).

In a standard instrumental contingency task, participants are asked to sample their actions and non-actions effectiveness in relation to control over the occurrence of an outcome (Wasserman et al., 1990). The action could be a simple button press 
and the outcome could be the discrete occurrence of an auditory or visual stimulus and these occur over a series of experimental trials (e.g., Msetfi et al., 2005). The experimenter can programme the objective contingency between action and outcome as measured by the normative metric, known as $\Delta \mathrm{P}$ (Allan, 1980), from and consider the four cells of a contingency table see Figure 1a. The table illustrates the type of events relevant for evaluating the relationship between Action and Outcome, the joint occurrence of the action and outcome (Cell A event), the presence of either the action or outcome alone (B or C) and the times that neither event occur (Cell D).

The contingency metric $\Delta \mathrm{P}$ is the difference between the conditional probabilities of the outcome given the presence and absence of the action, and provides a measure of the extent to which an action changes the likelihood of an outcome. Like a correlation coefficient, values for $\Delta \mathrm{P}$ vary from +1 , indicating complete control of the action over the outcome, through 0 , indicating that the presence of the action makes no difference to the occurrence of the outcome, through to -1 , indicating that the action prevents the outcome occurring.

After a series of experimental trials, during which the participant experiences specific contingency conditions like those shown in Figure 1b, the participant is asked to rate their control on a scale constructed to be isomorphic with the $\Delta \mathrm{P}$ metric. Bias or accuracy can be indicated by a comparison between the participant's rating and $\Delta \mathrm{P}$. However, further evidence comes from a comparison of a participant's ratings of two different conditions of the same contingency, but differing in terms of the number of actions, or the number of outcomes overall. This strategy has been used extensively to compare participant's judgements of two conditions with no contingency between action and outcome, but in which the rate or number of outcomes is manipulated (see 
Murphy, Vallee-Tourangeau, Msetfi \& Baker, 2005), and varying the temporal contiguity action-outcome (Vallee-Tourangeau, et al., 2005).

Experiments reported by Alloy and Abramson (1979) are an example of control ratings made about a specific uncontrollable condition that were used to infer the presence of a general sense of control. In their work, the authors described mildly depressed and non-depressed students who were asked to provide ratings of two conditions which had the same 0 value for $\Delta \mathrm{P}$ and thus participants had no control over the occurrence of a light flash outcome. There was however a different frequency (sometimes referred to as density) in the occurrence of outcomes across the two conditions. Non-depressed participants rated their control as higher in the high outcome condition than the low outcome condition, whereas depressed participants rated their control as low and similar across the two conditions. From these findings, it was suggested that non-depressed participants were overly optimistic, lured into perceiving control by the high outcome density, but the depressed participants were realistic in their judgements.

This interpretation of the judgements is based on the notion that bias and expectation have the strongest influence on people's ratings of control. Essentially the conditions of no control tested in this and numerous other studies are argued to fit with depressed people's negative expectations of having no control over events (e.g., Alloy, Abramson, \& Viscusi, 1981; Benassi \& Mahler, 1985; Martin, Abramson, \& Alloy, 1984; Msetfi, Murphy, \& Simpson, 2007; Msetfi, Murphy, Simpson, \& Kornbrot, 2005; Vasquez, 1987). These conditions set up a mismatch between the condition and non-depressed people's more positive expectations which lead the nondepressed to boost sense of control (Alloy \& Abramson, 1988; Alloy, Albright, Abramson, \& Dykman, 1990; Alloy \& Tabachnik, 1984). The assumption that 
findings from a specific situation relate to a generalised sense of control has been questioned (Skinner, 1996), but not directly tested. Generalised sense of control is usually measured psychometrically (e.g., Lachman \& Weaver, 1998), or with specific tasks which do not include a known control value (e.g., Thompson, Kent, Thomas, \& Vrungos, 1999) unlike the contingency tasks we have been describing.

Another approach is to assume that perceived control is data-driven. Thus, it has been argued that instead of, or perhaps in addition to, pre-existing biases being the main influencer of perceived control ratings, it is the processing of and learning about specific aspects of contingency experience (Blanco, Matute, \& Vadillo, 2009; 2011; 2012; Byrom, Msetfi, \& Murphy, 2015; Cavus \& Msetfi, 2016; Msetfi, Wade, \& Murphy, 2013) or the current motivational state of the participant (e.g., Baker et al., 2011). For example, Matute (1996) has argued that rates of behaviour, affect the degree of control perceived. Although participants might be instructed to sample actions and non-actions, there are variations in behaviour. Matute argued that nondepressed people are more likely to do this, and indeed there is evidence consistent with this position (Blanco et al., 2009, 2012).

It has also been argued that whilst behaviour might be affected by depression (or vice versa), depression and mood more generally might also affect the ability to process contingency information and integrate it into a person's perceptions of control (Msetfi et al., 2005). In particular, the nature of the one-way contingency (the $\Delta \mathrm{P}$ metric) implies that the rate that outcomes follow actions (cells A and B of the contingency matrix, Figure 1a) is compared to the rate of outcomes occurring in the absence of acting (i.e., the context shown in cells $\mathrm{C}$ and $\mathrm{D}$ of the matrix, Figure 1a). Msetfi et al.(2005), reported evidence suggesting that whereas non-depressed participants fully integrate non-action events (the context) outcome rates into their 
control ratings, which increases the level of control available to them, people scoring higher on a depression inventory do not though they can be trained to do so (Msetfi, Cavus, \& Brosnan, 2016a). For this reason, in this experiment we measured both ratings for the action and ratings for the context in which actions were performed

One aim of the current study was therefore to examine the link between specific condition control ratings and general control experience. In order to examine the effects of contingency experience, we constructed four contingency treatments (see Figure 1b), which each included four specific contingency conditions (four blocks, see block flowchart in Figure 1c). The treatments were constructed to test the effects of particular aspects of contingency experience, including the averaged $\Delta \mathrm{P}$ across groups (partial control $(\Delta \mathrm{P}=0.25)$ versus zero $(\Delta \mathrm{P}=0.0)$ and the effect of changing contingencies (Variable versus Constant) over blocks. In order to examine the effects of bias, we assumed that a rating made in advance of any specific treatment (i.e., pre rating) would represent biases brought into the experimental setting and we recorded ratings for the participant's action and their judgement of the context during the treatments. Then in order to measure a general sense of control based on accumulated experience, we asked participants to rate their perception of control after the treatment (post rating). In order to ensure the validity of the contingency experience and bias tests, we chose contingency task parameters which have been shown in previous work to produce accurate contingency ratings for all participants, whether regardless of depression score (Msetfi et al., 2013: Experiment 1). 
a

\begin{tabular}{|c|c|c|}
\hline \multicolumn{3}{|c|}{ Contingency table } \\
\hline & Outcome & Outcome \\
\hline Action & A & B \\
\hline Action & C & D \\
\hline
\end{tabular}

b Treatments' contingencies

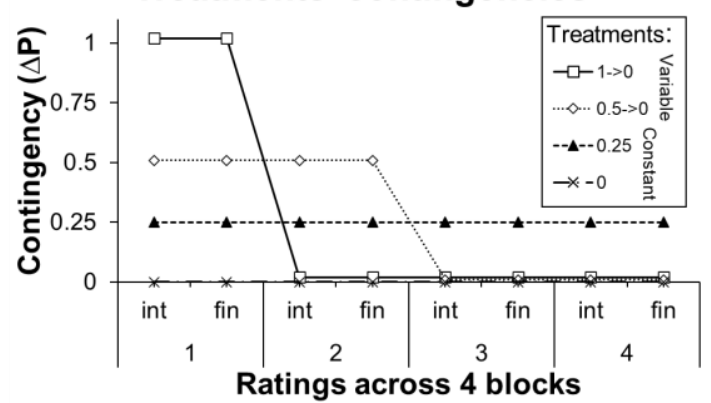

C

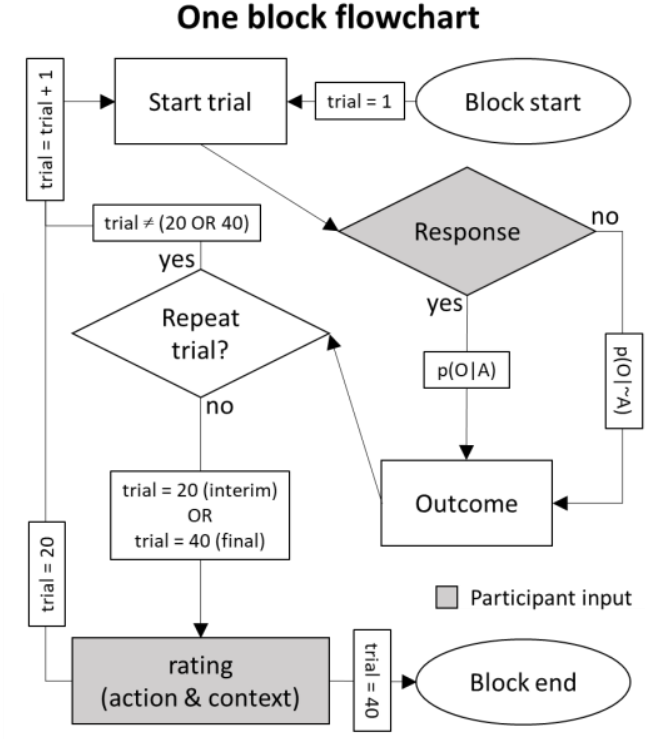

Figure 1. $\mathbf{a}$ contingency $2 \times 2$ table for Action (A) and Outcome (O); = absence of. $\mathbf{b}$ contingency experienced by every treatment group; contingency $(\triangle \mathrm{P})$ is calculated with the cells from the contingency table: $[\mathrm{A}(\mathrm{A}+\mathrm{B})]-$ $[\mathrm{C} /(\mathrm{C}+\mathrm{D})]$ or $\mathrm{p}($ Outcome|Action $)-\mathrm{p}($ Outcome| Action $)$; every block consisted on 40 trials, and four ratings were asked in two moments: one interim (int) and one at the end (fin) of each block $\mathbf{c}$ block flowchart, every block had 40 trials and every treatment had four blocks; two ratings moments occur per block: after trial 20 and after trial 40, i.e. at the end of the block. 


\section{Method}

\section{Participants}

Student volunteers were recruited using university email lists, social media sites, and Psychology Department online recruitment facilities. 210 participants attended appointments in the laboratory to participate. Participants were instructed to respond on some trials and not on others. Those that either almost always responded or never responded were removed from the analysis; on average $<15 \%$ or $>85 \%$ of trials. This is important because outliers in response behaviour can significantly alter the contingencies experienced by the participant (Matute, 1996), and our hypothesis depends on the participants experienced contingency. This exclusion criteria have been used in previous work (Msetfi, et al., 2014). For one participant, all data files were missing, and another 30 participants failed to complete the experimental task according to the instructions.

The final sample of 179 participants were $54 \%$ female and $46 \%$ male. During their visit to the laboratory, participants completed the Beck Depression Inventory (BDI) of depression symptoms and on the basis of these scores were categorised as low or high mood by scores on the Beck's Depression Index (Beck, Ward, Mendelson, Mock, \& Erbaugh, 1961). Low BDI, having no signs of depression, or high BDI, showing mild symptoms of depression (Low BDI $\leq 8, n=118$, Female $n=61$; High BDI $\geq 9, n=$ 61 , female $n=35$ ). Exclusions from the analysis mirrored the proportion of low and high BDI scores $(N=31,15 \%$; $\mathrm{n}=20$ Low BDI and $\mathrm{n}=10$ High BDI). The BDI groups were matched on gender, $\chi^{2}(1)=.32, p=.57$, as well as demographic and cognitive measures that might be expected to impact performance, including age, digit span, and estimated IQ (see Table 1). As expected, participants in the high BDI group 
showed greater evidence of depression, stress and anxiety than the low BDI group, although no differences in estimated IQ or working memory. 
Table 1

Characteristics of $\mathrm{n}=179$ included participants from $\mathrm{N}=210$ sample.

\begin{tabular}{|c|c|c|c|c|c|}
\hline Characteristic & Report & $\begin{array}{c}\text { Low BDI } \\
n=118\end{array}$ & $\begin{array}{c}\text { High BDI } \\
n=61\end{array}$ & Estimate & $\mathrm{p}$ \\
\hline Age & M (SD) & 24.35 (7.09) & $22.54(5.08)$ & $\mathrm{W}=3029.5$ & 0.08 \\
\hline Sex & female: \# (\%) & $61(52)$ & $35(57)$ & $X^{2}=0.31$ & 0.57 \\
\hline Handed & left: \# (\%) & $13(11)$ & $4(7)$ & $X^{2}=0.48$ & 0.49 \\
\hline Digit & M (SD) & $7.4(1.82)$ & $7.39(1.24)$ & $\mathrm{W}=3505.5$ & 0.77 \\
\hline Estimated IQ & $\mathrm{M}(\mathrm{SD})$ & $111.23(6.11)$ & $111.09(7.08)$ & $\mathrm{W}=3702.5$ & 0.75 \\
\hline $\mathrm{BDI}$ & $\mathrm{M}(\mathrm{SD})$ & $3.08(2.54)$ & $15.26(5.63)$ & $\mathrm{W}=7198$ & $<0.001$ \\
\hline DASS-A & $\mathrm{M}(\mathrm{SD})$ & $1.85(2.59)$ & $6.11(5.9)$ & $\mathrm{W}=5384.5$ & $<0.001$ \\
\hline DASS-D & $\mathrm{M}(\mathrm{SD})$ & $1.5(2.56)$ & 10.15 (7.09) & $\mathrm{W}=6869$ & $<0.001$ \\
\hline DASS-A & $\mathrm{M}(\mathrm{SD})$ & $3.69(3.96)$ & $11.79(7.8)$ & $\mathrm{W}=6110.5$ & $<0.001$ \\
\hline
\end{tabular}

Note: $\mathrm{M}=$ mean; $\mathrm{SD}=$ standard deviation; Low BDI $<9$ score, and High BDI $\geq 9$

score. DASS = Depression Anxiety Stress Scales, with subscales A = anxiety, D =

depression, $\mathrm{S}=$ stress; BDI = Beck Depression Inventory; W=Wilcoxon Rank Sum

test. Wilcoxon test was made because groups distributions did not meet t-test

assumptions (normality and homoscedasticity). 


\section{$\underline{\text { Design }}$}

This study is a mixed factorial design with mood (2: low BDI, high BDI) and treatment contingency group (4: Constant 0 , Constant 0.25 , Variable 1->0, Variable $.5->0)$ as between-subject factors. Participants for each treatment were exposed to different sequences of four blocks of contingencies, which are described in terms of $\mathrm{p}(\mathrm{O} \mid \mathrm{A}) / \mathrm{p}(\mathrm{O} \mid \sim \mathrm{A})$ (see Table 2, and Figure 1b). Two treatments received constant contingencies conditions (blocks) and two with variable contingencies. Treatment Constant 0.25 involved four blocks of 1.0/0.75 contingencies, a high outcome density zero contingency. Treatment Constant $\mathbf{0}$ served as the control treatment and experienced four blocks of $.75 / .75$ zero contingencies, with an average $\Delta \mathrm{P}=0$ (see Table 2). Treatment Variable 1->0 experienced one block of trials with total control 1.0/0 contingency, followed by three $.75 / .75$ zero contingency conditions and Treatment Variable .5->0 Zero experienced two positive 1.0/0.5 contingencies followed by two $.75 / .75$ zero contingencies. In three treatments (Constant 0.25 and the two Variable treatments), the overall contingency or average $\Delta P$ across the four blocks was same, (i.e., $\mathrm{DP}=.25$, see Figure $1 \mathrm{~b}$ ).

Participants control's ratings occurred before the treatment (pre) and after the treatment (post) for their action only, and at the middle (interim) and at the end (final) of each block for both their action and the context; every subject provided [2 (interim, final) $x 2$ (action, context) $\mathrm{x} 4$ (blocks) $]+2$ (pre, post for Action only) $=18$ ratings (see Figure 1b). The 'pre' rating was a measure of pre-existing sense of control or bias, whereas the 'post' rating was a measure of generalised sense of control following the 4 blocks. All control ratings were made on the same numeric scale with 
increments of $+/-1$, where +100 represented 'total control', 0 indicated 'no control' and -100 indicated 'preventative control'.

\section{$\underline{\text { Measures and Task }}$}

Measures. Participants completed the Beck Depression Inventory (BDI: Beck, et al., 1961) and the Depression Anxiety Stress Scales (DASS: Lovibond \& Lovibond, 1995), both of which are validated for use on student as well as patient samples.

Anxiety is comorbid with depression and has been related to contingency learning and learning about actions and contexts (e.g., Cohen et al. 2015), These BDI cut-offs for no depression (low BDI $=<9$ ) and greater evidence of depression (high BDI $>9$ ) have been used in previous studies (e.g., Alloy \& Abramson, 1979). In addition, participants completed the forwards version of the digit span task (Lezak, 1995) and provided demographic information, from which IQ was estimated (Barona, Reynolds, \& Chastain, 1984).

Contingency judgement task. An action-outcome contingency judgement task (see Msetfi et al., 2013) was used in this study. Each treatment was presented in a distinct context represented as a picture of a room displayed on the computer screen. The action was a press on the spacebar of the computer keyboard, which was active during a 3 second time window. The outcome was a 2 second auditory stimulus, which occurred or not depending on the programmed probability. Probabilities for $p(\mathrm{O} \mid \mathrm{A})$ and $p(\mathrm{O} \mid \sim \mathrm{A})$ were calculated separately across blocks of 8 trials to ensure the maximum possible accuracy of contingencies in relation to response rate variability. This resulted in two arrays of outcome / no outcome trials (e.g., 1, 1, 1, 0, 1, 1, 1, 0 for a $p=.75$ condition) the order of which was randomised over blocks of 8 trials. A unique sequence was generated for each participant. 
Each trial was separated from the next by a 3-s inter-trial interval (total time time $=8-\mathrm{s} ;$ these time parameters have produced accurate ratings regardless of BDI score in previous studies (Msetfi et al., 2013). Judgements were collected at the middle and at the end of each 40 trial block (see Figure 1c), after which the participant self-prompted to the next condition by pressing an onscreen button. Participants were asked to rate the amount of control that they perceived, Action ratings, as well as ratings of the context. The context was defined as other possible factors independent of their action that might be controlling the outcome (Context ratings). In addition to ratings, the programme (RealBasic, Macintosh operating systems), collected response rates (used to calculate $\mathrm{p}$ (response) per 20-trials segment within a block). 
Table 2

\begin{tabular}{|c|c|c|c|c|c|c|c|c|c|}
\hline Blocks & \multicolumn{2}{|c|}{$1 \mathrm{st}$} & \multicolumn{2}{|c|}{ 2nd } & \multicolumn{2}{|r|}{$3 r d$} & \multicolumn{2}{|r|}{ 4th } & \multirow{3}{*}{$\begin{array}{l}\text { overal } \\
\qquad \Delta \mathrm{P}\end{array}$} \\
\hline Contingency Experience & A & $\sim \mathrm{A}$ & A & $\sim \mathrm{A}$ & A & $\sim \mathrm{A}$ & A & $\sim \mathrm{A}$ & \\
\hline Condition & & & & & & $\Delta P_{3 r d}$ & & $\Delta P_{4 t h}$ & \\
\hline Variable $1->0(n=30)$ & 1.0 & 0.0 & .75 & .75 & .75 & .75 & .75 & .75 & \\
\hline $\mathrm{n}(\mathrm{BDI}$ high $)=15$ & & & & & 0.0 & & 0.0 & & \\
\hline Variable $0.5->0 \quad(n=32)$ & 1.0 & .50 & 1.0 & .50 & .75 & .75 & .75 & .75 & \\
\hline $\mathrm{n}(\mathrm{BDI}$ high $)=15$ & & & & & & 0.0 & & 0.0 & \\
\hline Constant $0.25(\mathrm{n}=27)$ & 1.0 & .75 & 1.0 & .75 & 1.0 & .75 & 1.0 & .75 & \\
\hline $\mathrm{n}(\mathrm{BDI}$ high $)=18$ & & & & & & 0.25 & & 0.25 & $.2 J$ \\
\hline Constant $0(n=29)$ & .75 & .75 & .75 & .75 & .75 & .75 & .75 & .75 & \\
\hline $\mathrm{n}(\mathrm{BDI}$ high $)=13$ & & & & & & 0.0 & & 0.0 & \\
\hline
\end{tabular}

Note: $\mathrm{A}=p(\mathrm{O} \mid \mathrm{A}) ; \sim \mathrm{A}=p(\mathrm{O} \mid \sim \mathrm{A}) ; \Delta P=\mathrm{A}-\sim \mathrm{A}$.

\section{Procedure}

On arrival in the laboratory and providing informed consent, participants completed the questionnaires and the digit span task (a measure of working memory). They then read the instructions for the contingency judgement task at which point the experimenter offered an opportunity for questions.

The causal cover story referred to a faulty household appliance which the participants were asked to evaluate. Rooms in a house are connected to a music system that includes speakers in each of the rooms, and a remote-control device with which participants should be able to control the music switching on in each of the 
rooms. The rooms and remote control were represented by pictures displayed on the computer screen. Residents of the house had previously complained that there may be a fault in the music system and that the participant's job was to test their own control over the music in each of the rooms. In each room, there would be numerous opportunities to test the music button on the remote control. Participants were instructed that they should try to equally sample actions and non-actions (the music might come on by itself). The task was completed in an individual experimental cubicle, and lasted approximately 20 minutes, after which the participant was debriefed.

\section{$\underline{\text { Statistical plan }}$}

We used linear mixed models (LMM, Bates, and Machler, 2014) in R (R Core Team, 2019), which are multilevel or hierarchical models, where in addition to including fixed effects (regressors or predictors in classical general linear models/multiple regressions) the model includes random effects given the nested structure of the data. In our case we included a random intercept for participants which were nested by treatments, and in addition blocks had a random intercept too. For detailed description of LMM parsimony see Bates et al., (2015). Firstly, to evaluate specific control, we built a full model (i.e., that includes all the possible experimental factors) to predict ratings (see Model Specification in Supplementary Materials). The regressors included in the full model were $i$. probability of response [p(response)], $i i$. within block rating (interim $=0$, final $=1), i i$. rating type $($ action $=0$, context $=1), i v$. group contingency dynamics (variable $=0$, constant $=1), v$. overall group contingency (zero $=0$, positive $=1), v i$. blocks ( 1 to 4$)$, and vii. high and low BDI score (low $=0$, high=1). All the possible second order interactions were included. After fitting the full 
model, we use a stepwise algorithm step() function (Kuznetsova, et al., 2017) to eliminate non predictive factors. Based on the factors that survived (i.e., were not eliminated) the stepwise pruning, we fitted the final model, which is the one presented in the results section (see the final model table in Supplementary Materials). We assumed a type 1 error rate $(\alpha)$ of 0.05 . Second, to evaluate general sense of control we used the same strategy but not including within block ratings and block as regressors and adding the pre and post factor.

To compare the final model versus our reduced models we used Bayes factors (BF) approximations (Wagenmakers, 2007). To increase robustness of the depression trait effect, we did a sensitivity analysis (see Higgins, \& Green, 2011) using BDI as a continuum normalizing the score (mean subtraction and divided by $\mathrm{SD}$, scale() $\mathrm{R}$ function used; see Sensitivity analysis in Supplementary Materials). We expected that both analyses would suggest a similar result.

R packages used: statistical analysis, lme4 and lmeTest (Bates, and Machler, 2014; Kuznetsova, et al.,2017); data processing, foreign, reshape2, plyr and dplyr ( $\mathrm{R}$ Core Team, 2018; Wickham, 2007; Wickham, 2011; Wickham, 2019a); models effect sizes and exporting to csv, psycho (Makowski, 2018); and data visualization, ggplot2 (Wickham, 2016), viridis (Garnier, 2018), and ggpubr (Kassambara, 2018). All the code used for this paper, including analysis and visualization is free to access on: https://github.com/santiagocdo/ContextControl.

\section{Results}

Participants' action and context control ratings (18 ratings per participant) for each of the four treatments separated by BDI groups are shown in Figure 2. These data suggest that ratings showed good discrimination between perfect, moderate and zero 
contingencies, with the variable groups' ratings changing in relation to the contingency whereas the groups receiving a constant contingency remaining relatively constant.

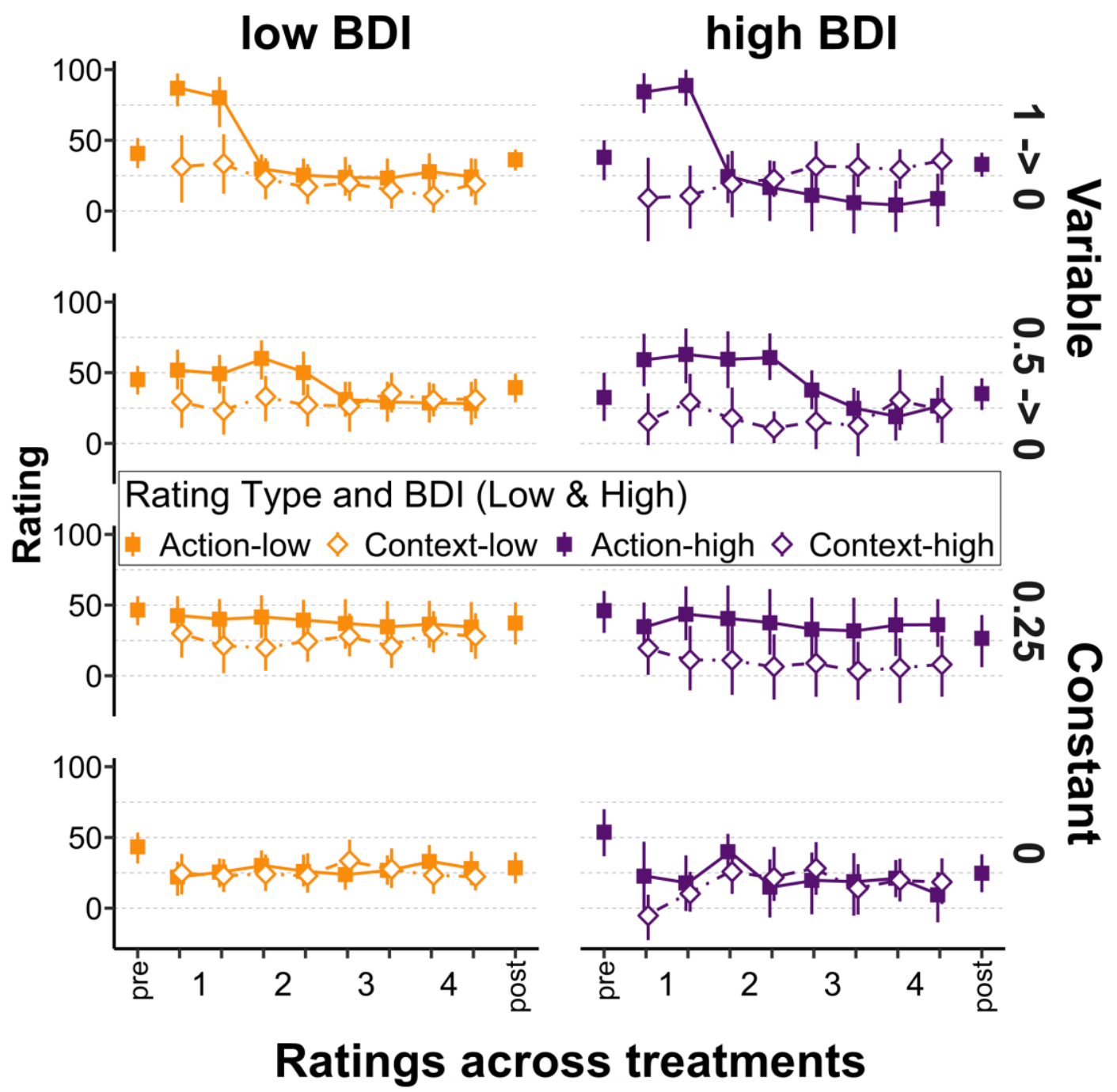

Figure 2. Ratings as a function of blocks ( 1 to 4 ) and ratings moments (interim or int, and final or fin). Pre and post ratings are included before and after the block started. The row subplots represent the treatments $(1->0=$ variable perfect $; 0.5->0=$ variable moderate $; 0.25=$ constant moderate $; 0=$ constant zero), and the column subplots are the low and high BDI groups. Fill squared are action ratings and empty diamond are context ratings. Error are a bootstrap 95\% confidence interval. 
GENERAL AND SPECIFIC PERCEIVED CONTROL 19 


\section{$\underline{\text { Specific sense of control }}$}

We fit the full model with the regressors and their $2^{\text {nd }}$ order interactions (see Model specification and Final model which includes a complete table of statistics in Supplementary Materials). After pruning the low predictive factors (Kuznetsova, et al., 2017), we obtained the final model which includes the regressors with predictive value for ratings.

We found main effects of probability of response $\beta=35.88$ [95\% CI from 25.15 to $46.61 ; \mathrm{Es}=0.16]$, Constant vs Variable treatments $\beta=-28.98$ [95\% CI from -38.42 to -19.54 , Es $=-0.13$ ], and a Block effect $\beta=-12.53$ [95\% CI from -15.35 to 9.7, Es $=-0.35]$. For a rating visualization, see Figure 2. There was no main effect on rating type nor BDI group however there was an interaction between BDI and ratings type (see below).

Regarding the interactions, rating type interacted with probability of response $\beta=-26.42$ [95\% CI from -39.69 to -13.14 , Es $=-0.12$ ], overall contingency $\beta=-8.1$ [95\% CI from -13.75 to -2.44 , Es $=-0.2$ ], block $\beta=7.23$ [95\% CI from 5.09 to 9.37 , Es $=0.2]$, and BDI group $\beta=-10.94$ [95\% CI from -16.00 to -5.87 , Es $=-0.27]$. In addition the difference between variable and constant groups interacted with block $\beta$ $=9.45[95 \% \mathrm{CI}$ from 7.30 to $11.59, \mathrm{Es}=0.26]$. This BDI $\mathrm{x}$ rating type interaction is consistent with previous observations. People who score high on BDI showed a difference in relative rating of their perceived control and the perceived by other factors (the context; Chase et al. 2011, Msetfi et al. 2016a). Furthermore, when we conducted a model comparison between the final model (deviance $=28451)$ and a BDI reduced model (deviance $=28471$; i.e., the final model without BDI factor), we found that the final model including BDI was a better fit, on the basis of a likelihood ratio, $\chi^{2}(2)=21.39, \mathrm{p}<0.001 ;$ and $\triangle \mathrm{AIC}=-17.39$ supporting the inclusion of BDI in the 
final model. Finally, we calculated a Bayes Factor $(\mathrm{BF})<0.1$ supporting again the final-BDI model. These results suggest that the categorical use of BDI provides a statistically significant better fit, by using three metrics for model comparisons. We also tested the use of BDI as a continuous variable, however, the sensitivity analysis is not consistent with the inclusion of BDI as a continuous variable in the analysis.

In summary, from Figure 2 we extract six results:(1) probability of response increase ratings overall; (2) constant (0.25 and 0) groups have lower overall ratings than variable $(1 \rightarrow 0$, and $0.5 \rightarrow 0)$; (3) ratings changed as a function of blocks; (4) the difference in the ratings type is larger for high BDI, only for the overall positive contingency treatments, but not for the zero constant contingency one. To explore this result we ran the Full model including the triple interaction between i) BDI group, ii) rating type, and iii) total contingency, but it was not significant. Nevertheless, as an exploratory post hoc analysis we ran two further similar models: the first including the treatments with overall positive contingencies (treatments: $1 \rightarrow 0,0.5 \rightarrow 0$, and 0.25 ) and the second only including the overall zero contingency (treatment: 0). For these two models we focused on the interaction between rating type and BDI group, this interaction was only significant for the positive overall contingencies $(\beta=-11.69$, $\mathrm{p}<0.001)$ and it was not significant for the 0 treatment $(\beta=-8.08, \mathrm{p}>0.05)$. (5) rating types difference are not the same across blocks; and (6) the rating type effect (i.e., the difference between action and context ratings) does not apply for the 0 contingency treatment (see lower treatment panel in Figure 2). On the other hand, Figure 3 shows: (1) high probability of response increase ratings (Figure 3a); and (2) rating type and probability of response interacts to predict ratings (Figure 3b). On the basis of these findings, and our previous reported studies (e.g., Msetfi, 2013, 2016a, 2016b, Byrom, et al., 2018), we explored the relation between Action and Context 
ratings as they varied as a function of probability of responding for the two BDI groups.

\section{Correlation between Ratings and Probability of Responding}

Figure 3 presents the correlation between ratings and probability of responding by 20trial segments within a block (this factor, interim vs final, was not significant in the rating model) and for the high and low BDI groups separately. We adjusted the $\mathrm{p}$ value with Bonferroni due to the 8 correlations (i.e., $0.05 / 8=0.00625$, see Figure $3 \mathrm{a}$ and 3b). In Figure 3a the analysis of the Low BDI participants found a significant correlation between action ratings and $\mathrm{p}$ (response), $\mathrm{r}(942)=0.21,95 \% \mathrm{CI}$ from 0.15 to $0.27, \mathrm{p}<0.00625$, and for context and $\mathrm{p}$ (response), $\mathrm{r}(942)=0.11,95 \% \mathrm{CI}$ from 0.05 to $0.17, \mathrm{p}<0.00625$. In contrast, the High BDI participants did not show significant correlations for context judgments and $\mathrm{p}($ response $), r(486)=0.01,95 \%$ CI from -0.07 to $0.1, p=0.76$, but a positive correlation between action judgments and $\mathrm{p}($ response $), \mathrm{r}(486)=0.16,95 \%$ CI from 0.07 to $0.24, \mathrm{p}<0.0065$. This interaction between context and depressive traits individuals has been found previously (e.g., Msetfi, et al., 2016b). For the Low BDI group, the difference between action minus context ratings was positively correlated with $\mathrm{p}($ response), $\mathrm{r}(942)=0.09,95 \% \mathrm{CI}$ from 0.02 to $0.15, \mathrm{p}<0.00625$ (not visualized). On the other hand, we found no correlation between action minus context rating difference and $\mathrm{p}$ (response) for the High BDI group $\mathrm{r}(486)=0.1,95 \%$ CI from 0.01 to $0.19, \mathrm{p}=0.018$ (not visualized). This correlational pattern between action-context and behaviour may suggest that the p(response) and ratings types are computed similarly for Low BDI participants and either differently for the High BDI group or as the figures suggest there is no relation between responding and ratings for the context. Finally, we report the correlations 
between action and context ratings for High and Low BDI groups. Low BDI ratings were significantly correlated, $r(942)=0.28,95 \%$ CI from 0.22 to $0.34, p<0.00625$; but not for High BDI, r $(486)=-0.018,95 \%$ CI from -0.1 to $0.07, p=0.7$ (see Figure $3 b)$.

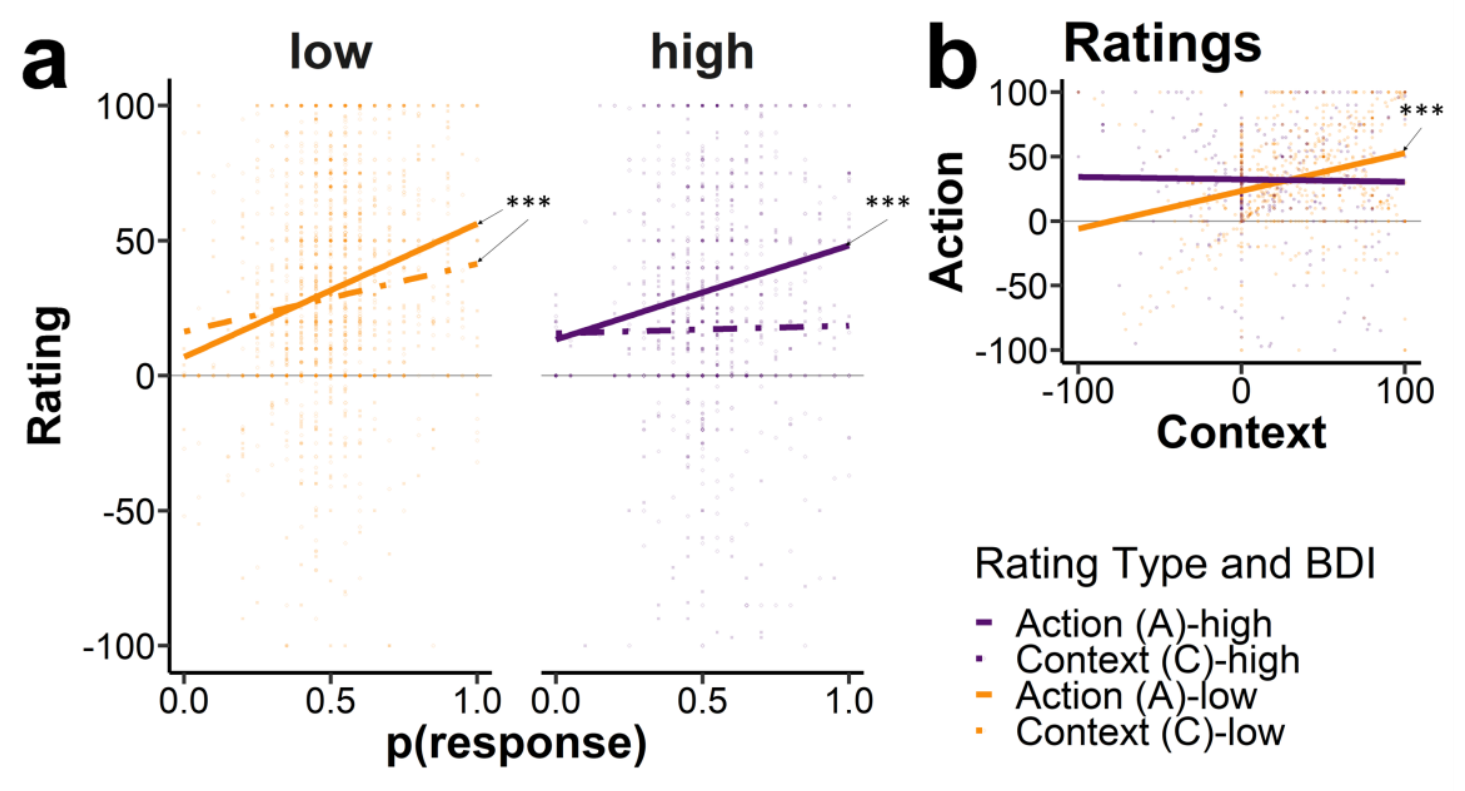

Figure 3. a subplot displays rating as a function of probability of response; for low and high BDI, and it display the linear regression line for Action (A; solid line) and for Context (C; dot dashed line) ratings. $\mathbf{b}$ subplot shows action rating as a function of context ratings. The colour signals low and high BDI. 


\section{General sense of control}

So far, we have discussed specific perceptions of control and sensitivity to probability of responding. One of our predictions was that participant's generalised sense of control would be reflected in their pre-existing sense of control and the average of all contingencies experienced. The generalised sense of control ratings were analysed using a similar method as the previous block ratings models. However, in this case we only included the pre and post ratings (and not the ratings during the 4 blocks). The full model had general sense of control ratings (pre and post), BDI (high and low), overall contingency (zero, positive), and variable-constant contingencies. After the model reduction (stepwise algorithm) we found a final model with the following regressors: rating moment (pre, post), variable-constant (variable, constant), and the interaction between the two. The only significant regressor that survived the stepwise factor pruning was the pre and post sense of control rating with $\beta=-10.94$ [95\% CI from -16.38 to -5.5 ; Es $=-0.37$, see the final model details in General sense of control Supplementary materials. This means that overall, irrespective of the treatment or BDI group participants' ratings were higher before the treatments than after the 4 blocks. This can be seen in Figure 2, when comparing the pre ratings estimates against the post rating estimates for any treatment or BDI group. This result suggests that participants may over-estimate their baseline control. Suggesting that pre-existing bias may have been a source of variability for judgments of experienced treatments, however we did not find evidence for this effect.

\section{Discussion}


In this experiment we tested the hypothesis that a generalised sense of agency and control is related to a combination of participant's pre-existing bias, the changes to the current contingency stream they are experiencing and, mood as measured by the Beck Depression Inventory. The key findings are: i) consistent with previous work, people irrespective of mood state, report ratings of contingency on the basis of their experience (i.e., they updated the specific sense of control), this sensitivity is often attributed to simultaneous perception of the effectiveness of actions and the effectiveness of the uncontrollable context (e.g, Msetfi et al., 2005; Murphy, Byrom $\&$ Msetfi, 2017); ii) ratings of action and context are differentially related to mood, we presented new evidence of the dissociable context and action processing reflected in behaviour, as evidenced by the interaction between rating type and BDI group suggests in the final model (Table S1). We now discuss these findings in relation to our original aims and in relation to theories and literature on perception of control related to depression, before acknowledging some caveats and also the limitations of this work.

One hypothesis for the relation between control ratings and mood has been that mood is related to a generalised or specific bias in processing of the contingencies. Some of our research has specifically tested how contingency learning emerges in participants focussing on the role that context plays in supporting learning about action (see Byrom, et al., 2015). An associative mechanism that supports contingency (e.g., Rescorla, \&Wagner, 1972) has been used to suggest that contingencies are acquired following a competitive or cooperative process that learns about the predictive stimulus properties of the Action (i.e., Response) and the properties of the context in which learning takes place (e.g., see Vallee-Tourangeau et al., 1998; 2005 for a theoretical discussion). In the current experiment, as with 
previous work, context learning specifically has been found to be a fundamental source of difference between people with varying evidence of depressive traits (Baker et al., 2011; Byrom et al., 2017; Msetfi, et al., 2005; 2007; 2009; 2013; 2016b). We hypothesized that perceptions of control related mood effects are fundamentally a disruption in context learning (Msetfi et al., 2005). We have previously demonstrated this in terms of context ratings using a task similar to the one presented here as well in experiments involving manipulation of the serotonin system (see Chase et al., 2011 and Msetfi, et al., 2016b). Here we found that people reporting lower mood showed a typicl action responding correlation but no correlation between ratings of context and responding.

This evidence for differential correlations between action and context ratings and probability of responding reported here for the High BDI group may be evidence in favour of a dissociation between self and context generated outcomes. This latter outcomes psychological are assumed to be generated by factors other than the self. And suggests that mood may be related to the maintenance of quite independent representations of outcome production. Other research has suggested that mood related biases are consistent with the maintenance of independent type of perceptual estimates (Pulcu, \& Browning, 2017). External locus of control, i.e., that events are produced by external forces instead of caused by the agent has been previously related to depressive symptoms (Johnson, \& Sarason, 1978; Hovenkamp-Hermelink, et al., 2019). This type of difference may make people showing signs of depression to be more sensitive to non-controllable adverse events (Johnson, \& Sarason, 1978). However, our evidence while correlational, suggests that the High BDI participants: a pre-existing context attentional bias produced more responses (Msetfi, et al., 2016); aor a general increased response rate enhanced overall control judgments, (Blanco, et 
al., 2012), particularly in High BDI participants where they over-integrate information from the context (Msetfi, et al., 2017).

It is interesting to note that these effects were most pronounced in the positive contingency treatments but not the Zero contingency treatment which is the usual contingency where depressive realism effects are shown. We did find differences in responding correlated with ratings for our high and low mood groups. These effects were found in the moderately positive contingency groups (Variable $1 \rightarrow 0$ and $.5 \rightarrow 0$ and Constant, .25) but not the constant 0 group. Depressive Realism effect which has generated a significant data base for mood related biases are reported for Zero contingencies (Allan et al., 2007; Alloy \& Abramson, 1979; Msetfi et al., 2005). This anomaly may suggest that previous work has been too focussed on uncorrelated action-outcome learning and overlooking differences at other correlation experiences.

Our perceived control has clear implications for our living (e.g. Keeton, et al., 2008) and not simply experience with uncorrelated events. The literature on depression (and non-depression) and perceived control is supported by an impressive body of quantitative experimental research. Beginning with Seligman's work on learned helplessness (Seligman \& Maier, 1967), and then Alloy and Abramson's (1979) depressive realism studies, as well as Langer's (1974) work on the illusion of control, the assumption has been that perceived uncontrollability and controllability, real or illusory, generalises from very specific situations to a generalised sense of control related to the whole of life. In the current study, we took a generalised measure of how much control participants expected to have (pre) and how much control they had at the end, experienced control (post). Our finding was that participants tend to overestimate control even when they do not have information of 
the contingency. However, the overestimation may be correct if the contingency had been highly positive, which was not the case (i.e., 0.25).

Furthermore, based on our experimental manipulation, there was no evidence that the experiences of individual contingencies 'averaged' to produce the generalised perception of control. Behavioural analysis approaches have been applied to these type of tasks to dissociate responding at the molecular level (trial level) and molar level (Overall treatment). Animals and humans respond in ways to reflect the dual action of these two sources of instrumental influence. Our data does not support evidence of responding based on a molar approach. Participants did not respond consistent with treating the individual treatments with similar overall contingency as similar. If this had been the case we might have expected to observe an interaction between pre and post with total overall contingency (0 vs 0.25$)$. However, the data suggests that participants adjusted their judgments in a molecular fashion (an online judgment adjusting given responses; for theoretical discussion between molar and molecular see: Malone, 2004, Sanabria, 2002).

We note that the notion of perceived control generalising from specific situations to a more generalised sense of control has been discussed previously (Skinner, 1996). Moreover, the literature on generalised sense of control has developed largely separately from literature involving specific, known contingency situations, with psychometric measures being the preferred measurement option for generalised control (Friedland et al., 1992; Lachman \& Weaver, 1998; Paulhus, 1983; Thompson et al., 1999). Our findings are consistent with the development of these distinct, yet related, lines of research, and with the idea that the generalised sense of control is orthogonal to specific situational control. However, memory research has shown that two strategies guide judgments (Aust, et al., 2019); and these two 
strategies may be modulating the sense of control. Another proposition strongly evident in the literature is that assessments of individual contingency situations are a function of pre-existing bias as well as the contingency itself (Alloy \& Tabachnik, 1984). However, our findings showed that pre-existing bias had little impact on specific control ratings. The only significant effect on these ratings was the contingency programmed at a particular block, which is again consistent with the idea that generalised and specific control are distinct (as expected and retrieved are different mental events, see Jozefowiez, 2018). In this study, scoring higher on a depression inventory was not related to either type of control. Though, we note that contextual factors (Msetfi et al., 2016) and behaviour variation (Blanco et al., 2009; 2011) are known to influence effects mediated by mood and depression, indeed here we demonstrate a differential de-coupling of behaviour and judgements in our two groups(see also, Na, et al., 2019).

Pre existing mood differences, measured on the day of test, did not predict generalised sense of control. This finding suggests that fluctuations in depression levels on a daily or weekly basis, as measured by the Beck Depression Inventory (Beck et al., 1961), may not impact these specific and generalised one session task perceptions. According to Beck's cognitive theory of depression, such generalised perceptions are reflections of long-held schema, which in the case of depression are rigid and inflexible (Clark, Beck, \& Alford, 1999). Our findings are generally consistent with this cognitive conceptualisation of depression. Evidence also shows that depression can affect situation specific control judgements, but this is dependent on the type of data available on which to base the control judgement (Msetfi et al., 2005), suggesting that specific control judgements are based on basic perception and 
learning experience (i.e., are data-driven), rather than higher level depressive cognitions.

Process theories have been applied to understand the development of underlying control or contingency learning. For example, the Rescorla-Wagner model (RWM: Rescorla \& Wagner, 1972) is an associative learning theory, which has been applied to contingency learning (e.g., Baker et al., 1996; Dickinson, Shanks, \& Evenden, 1984; Vallee-Tourangeau et al., 1998) as well as learning of contingencies in animals. The RWM describes how associations develop between cues or actions and subsequent outcomes when they are paired, and how other stimuli (the context) also become associated with the same outcomes. The strength of the action-outcome association relative to the context is the index on which perceived control ratings are based. An alternative model, which does not specify a process but does specify the type of information relevant to a control judgment, is the $\Delta P$ model. Whilst for $\Delta P$ is a measure of a contingency in a given situation, it is also a measure of the control a person 'should' perceive if they behave like a rational statistical or mathematical rule and combine information in a similar manner. $\Delta P$ produces similar predictions to RWM when all events are weighted equally (Baker, Murphy Vallee-Tourangeau, 1996). The suggestion that perceptions of control emerge from this type of mechanism is consistent with the theory that disruption in mood is related to a breakdown in the normative assignment of control as it relates to causal effectiveness.

\section{Conclusions}

Individual differences in how people learn has implications for diagnostic criterion for psychopathology (e.g., Murphy \& Msetfi 2014; Murphy, Msetfi and Byrom, 2017). In this study we investigated the relationship between specific and general control experiences, and the extent to which these are affected by pre-existing bias and mood as measured using a depression inventory as well as the role that 
behavioural control contributed to judgments. Our data suggest that specific and general control are distinct aspects of the sense of control. Perceptions of specific control situations were based on the contingencies people to which they were exposed. The likelihood of responding and ratings that were provided after behaviour were predictive of mood group. 


\section{Acknowledgements}

SC thanks to the University of Guadalajara for his PhD scholarship. We also acknowledge to the two anonymous reviewers for very useful comments and suggestions. Funding for this research was provided by ESRC UK to R.M and R.A.M. RES-062-23-2525. 


\section{References}

Abramson, L. Y., Metalsky, G. I., \& Alloy, L. B. (1989). Hopelessness depression: A theory based subtype of depression. Psychological Review, 96, 358-372.

Ackermann, R., \& DeRubeis, R. J. (1991). Is depressive realism real? Clinical Psychology Review, 11(5), 565-584.

Allan, L. G. (1980). A note on measurement of contingency between two binary variables in judgment tasks. Bulletin of the Psychonomic Society, 15(3), 147149.

Allan, L. G. (1993). Human contingency judgments: Rule-based or associative? Psychological Bulletin, 114(3), 435-448.

Allan, L. G., Siegel, S., \& Hannah, S. (2007). The sad truth about depressive realism. The Quarterly Journal of Experimental Psychology, 60(3), 482-495.

Alloy, L. B., \& Abramson, L. Y. (1979). Judgement of contingency in depressed and non-depressed students: Sadder but wiser? Journal of Experimental Psychology-General, 108(4), 441-485.

Alloy, L. B., \& Abramson, L. Y. (1988). Depressive realism: Four theoretical perspectives. In L. B. Alloy (Ed.), Cognitive processes in depression (pp. 223265). New York, NY, US: Guilford Press.

Alloy, L. B., Abramson, L. Y., \& Viscusi, D. (1981). Induced mood and the illusion of control. Journal of Personality and Social Psychology, 41(6), 1129-1140. doi:10.1037/0022-3514.41.6.1129

Alloy, L. B., Albright, J. S., Abramson, L. Y., \& Dykman, B. M. (1990). Depressive realism and nondepressive optimistic illusions: The role of the self. In R. E. Ingram (Ed.), Contemporary psychological approaches to depression: Theory, research, and treatment (pp. 71-86). New York, NY, US: Plenum Press.

Alloy, L. B., \& Tabachnik, N. (1984). Assessment of covariation by humans and animals: The joint influence of prior expectations and current situational information. Psychological Review, 91(1), 112-149.

Aust, F., Haaf, J. M., \& Stahl, C. (2019). A memory-based judgment account of expectancy-liking dissociations in evaluative conditioning. Journal of Experimental Psychology: Learning, Memory, and Cognition, 45(3), 417.

Baker, A.G. \& Msetfi, R.M., N. Hanley and Murphy, R.A., (2011). Sadly not wiser. In L.Hogarth \& M.Hazelgrove (Eds.) Clinical applications of learning theory. Psychology Press.

Baker, A.G., Murphy, R.A.\& Vallée-Tourangeau, F. (1996). Associative and normative models of causal induction: Reacting to versus understanding cause. In D.R.Shanks, K.J.Holyoak \& D.L.Medin (Eds.), The Psychology of Learning and Motivation, Vol. 34, (pp. 1-45) San Diego, CA: Academic Press.

Barona, A., Reynolds, C. R., \& Chastain, R. (1984). A demographically based index of premorbid intelligence for the WAIS-R. Journal of Consulting and Clinical Psychology, 52(5), 885-887. doi:10.1037/0022-006x.52.5.885

Bates, D., \& Machler, M. (2014). Package Lme4: Linear Mixed-Effects Models Using Eigen and S4. Journal of statistical software.

Bates, D., Kliegl, R., Vasishth, S., \& Baayen, H. (2015). Parsimonious mixed models. arXiv preprint arXiv:1506.04967. 
Beck, A. T., Ward, C. H., Mendelson, M., Mock, J., \& Erbaugh, J. (1961). An inventory for measuring depression. Archives of General Psychiatry, 4, 561571.

Benassi, V. A., \& Mahler, H. I. M. (1985). Contingency judgements by depressed college students: Sadder but not always wiser. Journal of Personality and Social Psychology, 49(5), 1323-1329.

Blanco, F., Matute, H., \& Vadillo, M. A. (2009). Depressive realism: Wiser or quieter? The Psychological Record, 59(4), 551-562.

Blanco, F., Matute, H., \& Vadillo, M. A. (2011). Making the uncontrollable seem controllable: The role of action in the illusion of control. The Quarterly Journal of Experimental Psychology, 64(7), 1290-1304. doi:10.1080/17470218.2011.552727

Blanco, F., Matute, H., \& Vadillo, M. A. (2012). Mediating role of activity level in the depressive realism effect. PLoS ONE, 7(9). doi:10.1371/journal.pone.0046203

Byrom, N., Msetfi, R.M. Murphy, R.A. (2018). Human latent inhibition: Problems with the stimulus exposure effect. Psychonomic Bulletin \& Review, 25,21022118.

Byrom, N.C., Msetfi, R.M., \& Murphy, R.A. (2015). Two pathways to causal control: use and availability of information in the environment in people with and without signs of depression. Acta Psychologica, 157, 1-12. doi:10.1016/j.actpsy.2015.02.0

Byrom, N. \& Murphy, R.A. (2018). Individual differences are more than a gene $\mathrm{x}$ environment interaction: The role of learning. Journal Experimental Psychology: Animal Learning \& Cognition, 44, 36-55.

Cavus, H. A., \& Msetfi, R. M. (2016). The Effects of Cognitive Load during Intertrial Intervals on Judgements of Control: The Role of Working Memory and Contextual Learning. Acta Psychologica, 171, 47-56.

Chase, H., Crockett, M.J., Msetfi, R.M., Murphy, R.A., Clark, L., Sahakian, B.J. \& Robbins, T.W. (2011) 5-HT Modulation by Acute Tryptophan Depletion of Human Instrumental Contingency Judgements depends on Baseline Depression Score. Psychopharmacology, 213, 615-623.

Clark, D. A., Beck, A. T., \& Alford, B. A. (1999). Scientific foundations of cognitive theory and therapy of depression. New York, NY, US: John Wiley and Sons, Inc.

Cohen-Kadosh, K, Haddad, A.D.M., Heathcote, L. Murphy, R.A., Pine, D. \& Lau, J. (2015). High trait anxiety during adolescence interferes with discriminatory context and learning. Neurobiology of Learning and Memory, 123, 50-57.

Dickinson, A., Shanks, D., \& Evenden, J. (1984). Judgment of act-outcome contingency: The role of selective attribution. Quarterly Journal of Experimental Psychology Section a-Human Experimental Psychology, 36(1), 29-50.

Dobson, K., \& Franche, R. L. (1989). A conceptual and empirical review of the depressive realism hypothesis. Canadian Journal of Behavioural ScienceRevue Canadienne Des Sciences Du Comportement, 21(4), 418-433.

Folkman, S. (1984). Personal control and stress and coping processes: A theoretical analysis. Journal of Personality and Social Psychology, 46(4), 839-852. doi:10.1037/0022-3514.46.4.839 
Friedland, N., Keinan, G., \& Regev, Y. (1992). Controlling the uncontrollable: effects of stress on illusory perceptions of controllability. Journal of Personality and Social Psychology, 63(6), 923.

Garnier, S., (2018). viridis: Default Color Maps from 'matplotlib'. R package version 0.5.1. https://CRAN.R-project.org/package=viridis

Griffiths, O., Shehabi, N., Murphy, R.A. \& Le Pelley, M.E. (2019). Superstition predicts perception of illusory control. British Journal of Psychology, 110, 499-518.

Hovenkamp-Hermelink, J. H., Jeronimus, B. F., Spinhoven, P., Penninx, B. W., Schoevers, R. A., \& Riese, H. (2019). Differential associations of locus of control with anxiety, depression and life-events: A five-wave, nine-year study to test stability and change. Journal of affective disorders, 253, 26-34.

Higgins, J. P., \& Green, S. (Eds.). (2011). Cochrane handbook for systematic reviews of interventions (Vol. 4). John Wiley \& Sons.

Johnson, J. H., \& Sarason, I. G. (1978). Life stress, depression and anxiety: Internalexternal control as a moderator variable. Journal of psychosomatic research, 22(3), 205-208.

Jozefowiez, J. (2018). Associative versus predictive processes in Pavlovian conditioning. Behavioural processes, 154, 21-26.

Keeton, C. P., Perry-Jenkins, M., \& Sayer, A. G. (2008). Sense of control predicts depressive and anxious symptoms across the transition to parenthood. Journal of family psychology, 22(2), 212.

Kassambara, A., (2018). ggpubr: 'ggplot2' Based Publication Ready Plots. R package version 0.2. https://CRAN.R-project.org/package $=$ ggpubr

Kuznetsova, A., Brockhoff, P. B., \& Christensen, R. H. B. (2017). lmerTest package: tests in linear mixed effects models. Journal of Statistical Software, 82(13).

Lachman, M. E., \& Weaver, S. L. (1998). The sense of control as a moderator of social class differences in health and well-being. Journal of Personality and Social Psychology, 74(3), 763.

Langer, E. J. (1974). The illusion of control. (35), ProQuest Information \& Learning, US. Retrieved from http://search.ebscohost.com/login.aspx?direct=true\&db=psyh\&AN=197702492-001\&site=ehost-live Available from EBSCOhost psyh database.

Lezak, M. D. (1995). Neuropsychological assessment (3rd ed.). New York: Oxford University Press.

Lovibond, P. F., \& Lovibond, S. H. (1995). The structure of negative emotional states: Comparison of the Depression Anxiety Stress Scales (DASS) with the Beck Depression and Anxiety Inventories. Behaviour Research and Therapy, 33(3), 335-343.

Makowski, D. (2018). The Psycho Package: An Efficient and Publishing-Oriented Workflow for Psychological Science. Journal of Open Source Software, 3(22), 470. Available from https://github.com/neuropsychology/psycho.R

Malone, J. C. (2004). Modern molar behaviorism and theoretical behaviorism: Religion and science. Journal of the Experimental Analysis of Behavior, 82(1), 95-102.

Martin, D. J., Abramson, L. Y., \& Alloy, L. B. (1984). Illusion of control for self and others in depressed and non-depressed college students. Journal of Personality and Social Psychology, 46(1), 125-136. 
Matute, H. (1996). Detecting response-outcome independence in analytic but not in naturalistic conditions. Psychological Science, 7(5), 289-293. doi:10.1111/j.1467-9280.1996.tb00376.x

Msetfi, R. M., Cavus, H. A., \& Brosnan, L. (2016a). Enhanced Attention to Context Increases Perceived Control in Mild Depression. Quarterly Journal of Experimental Psychology, 69(6), 1073-1081. doi:10.1080/17470218.2016.1138134

Msetfi, R.M., Kumar, P., Harmer C. \& Murphy, R.A (2016b). SSRI enhances sensitivity to background outcomes and modulates response rates: A randomized double blind study of instrumental action and depression, Neurobiology of Learning and Memory, 131, 76-82.

Msetfi, R. M., Byrom, N., \& Murphy, R. A. (2017). To neglect or integrate contingency information from outside the task frame, that is the question! Effects of depressed mood. Acta psychologica, 178, 1-11.

Msetfi, R. M., Kornbrot, D. E., Matute, H., \& Murphy, R. A. (2015). The relationship between mood state and perceived control in contingency learning: effects of individualist and collectivist values. Frontiers in Psychology, 6.

Msetfi, R.M., Murphy, R.A. and Kornbrot, D. (2012). Effect of mild depression on time discrimination. Quarterly Journal of Experimental Psychology. 65, 632645.

Msetfi, R. M., Murphy, R. A., \& Simpson, J. (2007). Depressive realism and the effect of intertrial interval on judgements of zero, positive, and negative contingencies. The Quarterly Journal of Experimental Psychology, 60(3), 461481.

Msetfi, R. M., Murphy, R. A., Simpson, J., \& Kornbrot, D. E. (2005). Depressive realism and outcome density bias in contingency judgements: The effect of context and the inter-trial interval. Journal of Experimental Psychology: General, 134(1), 10-22.

Msetfi R.M., Murphy R.A. Simpson, J. \& Kornbrot,D. (2009). Impaired context maintenance in mild to moderately depressed students. Quarterly Journal of Experimental Psychology, 62, 653-662.

Msetfi, R. M., Wade, C., \& Murphy, R. A. (2013). Context and time in causal learning: Contingency and mood dependent effects. PLoS ONE, 8(5), e64063. doi:10.1371/journal.pone.0064063

Murphy, R.A., Byrom, N. \& Msetfi, R. M. (2017). The problem with explaining symptoms: The origin of biases in causal processing, European Journal for Person Centered Healthcare, 5, 344-350

Murphy, R.A., Vallée-Tourangeau, F., Msetfi, R.M. \& Baker, A.G. (2005). Signaloutcome contingency, contiguity and the depressive realism effect. In A.Wills (Ed.) New directions in associative learning. (Ch. 10) Hillsdale, NJ: Lawrence Erlbaum Associates.

Murphy, R.A. \& Msetfi, R.M. (2014). Individual differences and associative learning. Frontiers in Psychology: Individual Differences, doi: 10.3389/fpsyg.2014.00466

Na, S., Chung, D., Jung, J., Hula, A., Fiore, V. G., Dayan, P., \& Gu, X. (2019). Humans use forward thinking to exert social control. bioRxiv doi: https://doi.org/10.1101/737353

Paulhus, D. (1983). Sphere-specific measures of perceived control. Journal of Personality and Social Psychology, 44(6), 1253.

Pulcu, E., \& Browning, M. (2017). Affective bias as a rational response to the statistics of rewards and punishments. Elife, 6, e27879. 
Rescorla, R., \& Wagner, A. (1972). A theory of Pavlovian conditioning: Variations in the effectiveness of reinforcement and non-reinforcement. In A. Black \& W. Prokasy (Eds.), Classical conditioning II: Theory and research (pp. 64-99). New York: Appleton Century Crofts.

R Core Team (2018). foreign: Read Data Stored by 'Minitab', 'S', 'SAS', 'SPSS', 'Stata', 'Systat', 'Weka', 'dBase', .... R package version 0.8-71. https://CRAN.Rproject.org/package=foreign

R Core Team (2019). R: A language and environment for statistical computing. $\mathrm{R}$ Foundation for Statistical Computing, Vienna, Austria. URL https://www.Rproject.org/

Sanabria, F. (2002). Análisis molar y molecular: dos visiones de la conducta. Universitas Psychologica, 1(2), 27-33.

Seligman, M. E. (1975). Helplessness: On development, depression and death. New York: W. H. Freeman and Company.

Seligman, M. E., \& Maier, S. F. (1967). Failure to Escape Traumatic Shock. Journal of Experimental Psychology, 74(1), 1-9.

Skinner, E. A. (1996). A guide to constructs of control. Journal of Personality and Social Psychology, 71(3), 549-570. doi:doi:10.1037/0022-3514.71.3.549

Thompson, S. C., Kent, D. R., Thomas, C., \& Vrungos, S. (1999). Real and Illusory Control Over Exposure to HIV in College Students and Gay Men1. Journal of Applied Social Psychology, 29(6), 1128-1150.

Vasquez, C. (1987). Judgement of contingency: Cognitive biases in depressed and nondepressed subjects. Journal of Personality and Social Psychology, 52(2), 419-431.

Vallee-Tourangeau, Murphy, R.A. \& Baker, A.G. (2005). Contiguity and the Outcome Density Bias in Action-Outcome Contingency Judgments, Quarterly Journal of Experimental Psychology: Comparative and Physiological, 58B, 177-192.

Vallée-Tourangeau, F., Murphy, R.A., Drew, S. \& Baker, A.G. (1998). Judging the importance of constant and variable candidate causes: A test of the Power PC theory. Quarterly Journal of Experimental Psychology, 51A, 65-84.

Wagenmakers, E. J. (2007). A practical solution to the pervasive problems of $\mathrm{p}$ values. Psychonomic bulletin \& review, 14(5), 779-804.

Wickham, H. (2007). Reshaping Data with the reshape Package. Journal of Statistical Software, 21(12), 1-20. URL http://www.jstatsoft.org/v21/i12/.

Wickham, H. (2011). The Split-Apply-Combine Strategy for Data Analysis. Journal of Statistical Software, 40(1), 1-29. URL http://www.jstatsoft.org/v40/i01/.

Wickham, H., François, R., Henry, L., and Müller, K., (2019). dplyr: A Grammar of Data Manipulation. R package version 0.8.0.1. https://CRAN.Rproject.org/package $=$ dplyr

Wickham, H., (2016). ggplot2: Elegant Graphics for Data Analysis. Springer-Verlag New York. 